\title{
Contrail cirrus supporting areas in model and observations
}

\author{
Ulrike Burkhardt, ${ }^{1}$ Bernd Kärcher, ${ }^{1}$ Michael Ponater, ${ }^{1}$ Klaus Gierens, ${ }^{1}$ \\ and Andrew Gettelman ${ }^{2}$ \\ Received 31 March 2008; revised 14 June 2008; accepted 25 June 2008; published 23 August 2008.
}

[1] Contrails form and persist dependent on the surrounding moisture, temperature and pressure fields and on fuel and aircraft specific variables. After formation, contrail persistence requires only supersaturation relative to ice. The fractional area in which contrails can form is called potential contrail coverage. We introduce a potential contrail cirrus coverage equivalent to the cloud free supersaturated area. This field, simulated by the ECHAM4 climate model, agrees fairly well with estimates of supersaturation frequency as inferred from aircraft and satellite measurements. In areas where the two potential coverages are different, especially at lower flight levels, potential contrail coverage is not a valid estimate of maximum attainable contrail cirrus coverage. We parameterize both potential coverages consistently with the ECHAM4 cloud cover parameterization. A comparison of the potential contrail coverage with an earlier estimate reveals substantial differences especially at upper height levels in the tropics. Citation: Burkhardt, U., B. Kärcher, M. Ponater, K. Gierens, and A. Gettelman (2008), Contrail cirrus supporting areas in model and observations, Geophys. Res. Lett., 35, L16808, doi:10.1029/2008GL034056.

\section{Introduction}

[2] Contrails form in sufficiently cold and moist air (Schmidt-Appleman criterion, SAC) [Schumann, 1996], at temperatures below $\sim 233 \mathrm{~K}$ and pressures below $300 \mathrm{hPa}$, and persist when air is supersaturated relative to ice. When persistent, contrails can spread into contrail cirrus eventually losing their line shape. Contrail cirrus change the radiative balance of the atmosphere. Their radiative effect may be larger than that due to the (accumulated) aircraft $\mathrm{CO}_{2}$ emissions [Sausen et al., 2005].

[3] Current contrail cirrus modeling efforts using general circulation models (GCMs) are restricted to line-shaped contrails. Estimates of contrail coverage rely on the introduction of a potential contrail coverage [Sausen et al., 1998; Ponater et al., 2002] describing the fractional area of the atmosphere that is susceptive to persistent contrail formation. It has therefore been interpreted as the maximum possible contrail coverage. Potential contrail coverage has also been widely used in offline estimates of global contrail radiative forcing [e.g., Minnis et al., 1999; Myhre and Stordal, 2001; Stuber and Forster, 2007; Rädel and Shine, 2008].

\footnotetext{
${ }^{1}$ Deutsches Zentrum für Luft- und Raumfahrt, Institut für Physik der Atmosphäre, Oberpfaffenhofen, Germany.

${ }^{2}$ National Center for Atmospheric Research, Boulder, Colorado, USA.
}

Copyright 2008 by the American Geophysical Union. 0094-8276/08/2008GL034056\$05.00
[4] Our work is motivated by the fact that potential contrail coverage is not the upper bound for contrail cirrus coverage since it reflects the formation conditions (SAC) for persistent contrails. Once formed, contrails may persist at times or be advected into areas, in which the SAC is not met. Therefore, we propose in section 2 the definition of two distinct potential coverages, potential contrail and potential contrail cirrus coverage. The former describes the fractional area in which contrails can form (according to the SAC) and persist and the latter the fractional area in which contrail cirrus can persist once they have been formed. Another motivation for the paper is the inconsistency between the current parameterization of potential contrail coverage [Ponater et al., 2002; Sausen et al., 1998] and the assumptions underlying the relative humidity based parameterization of cloud coverage [Sundqvist, 1978]. We introduce a modified parameterization of potential contrail coverage that is consistent with the cloud scheme and compare our results to the earlier ones. In section 3 we compare with in situ (MOZAIC - Measurements of ozone and water vapour by Airbus in-service aircraft) [Gierens et al., 1999] and satellite estimates (AIRS - Atmospheric Infrared Sounder) [Gettelman et al., 2006] of supersaturation to evaluate the potential contrail cirrus parameterization.

\section{Model and Methods}

[5] We use the ECHAM4 climate model [Roeckner et al., 1999] at T30 resolution with 39 levels [Land et al., 2002]. As in most other GCMs, ice supersaturation is not resolved on the grid scale. The diagnostic cloud cover parameterization in ECHAM4 is based on the grid mean relative humidity [Sundqvist, 1978]. The parameterization makes assumptions about the subgrid scale variability of total water, so that natural cloud coverage, $b_{c i}$, is given by:

$$
b_{c i}=1-\sqrt{1-\frac{r-r_{c i}}{r_{s a t}-r_{c i}}} .
$$

Cloud coverage (Figure 1, black curve) is larger than zero when $r$ exceeds the critical relative humidity for natural cloud formation, $r_{c i}$. When relative humidity reaches saturation, $r_{\text {sat }}=1$, full cloud coverage is realized. Equation 1 assumes a uniform probability density function (PDF) of total (vapor plus condensate) water mixing ratio with fixed variance [Gregory et al., 2002] (see also auxiliary material $^{1}$ ). Simulated cloud coverage has been validated by Chen and Roeckner [1997]. In the following we will only

\footnotetext{
${ }^{1}$ Auxiliary materials are available in the HTML. doi:10.1029/ 2008 GL034056.
} 


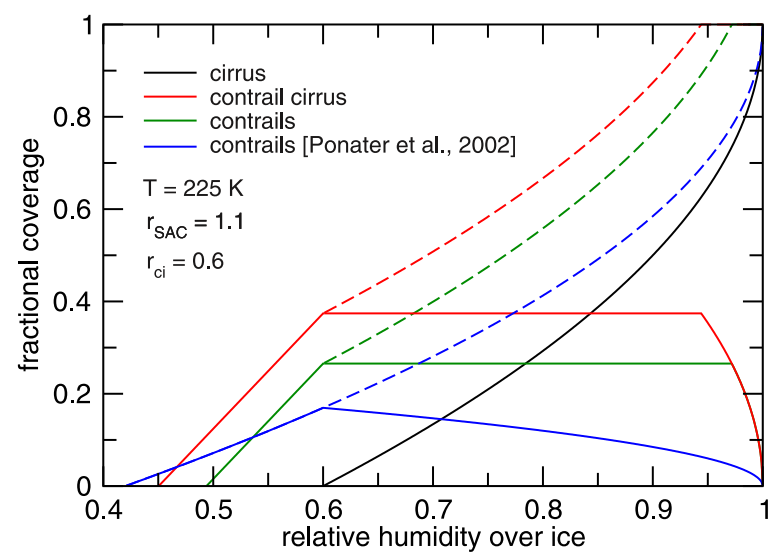

Figure 1. Cirrus coverage $b_{c i}$ (black), potential contrail coverage (solid green), potential contrail cirrus coverage $B_{c c}$ (solid red) and potential contrail coverage according to Ponater et al. [2002] (solid blue) versus grid mean relative humidity over ice $r$ at $T=225 \mathrm{~K}, r_{c i}=0.6$ and $r_{S A C}=1.1$. Dashed curves indicate the sums of $b_{c i}$ and the respective potential coverage; when $r<r_{c i}$ dashed and solid curves coincide.

discuss clouds at or above $300 \mathrm{hPa}$ where temperatures are such $(<241 \mathrm{~K})$ that only in the tropics model clouds contain few supercooled water droplets $(<5 \%)$. Hence, all $r$-values refer to the ice phase.

[6] In nature contrails and contrail cirrus develop and persist in ice-supersaturated air masses whereas natural cirrus predominantly forms in the upper troposphere at high supersaturations via homogeneous freezing [e.g., Jensen et al., 2001], and can then persist in air that is at least saturated. Analogous to the cloud coverage, fractional supersaturated area has to be parameterized. We calculate a proxy for the fractional supersaturated area consistent with the existing cloud cover parameterization using the same total water PDF as used for natural cirrus but a different critical relative humidity, $r_{c c}$. The latter indicates the value of $r$ (grid mean) at which a part of the model grid box is at least ice-saturated. The fact that natural cirrus is mostly initiated at substantial ice supersaturation (at the homogeneous freezing threshold $r_{\text {nuc }}=2.349-T[\mathrm{~K}] / 259$ ranging between 1.5 and 1.7 [Koop, 2004], which is below liquid water saturation) motivates a grid mean critical relative humidity for supersaturation, $r_{c c}$,

$$
\frac{r_{c c}}{r_{c i}}=\frac{r_{\text {sat }}}{a \cdot r_{n u c}}=\frac{1}{a \cdot r_{n u c}},
$$

with $a \leq 1$ accounting for the fact that cirrus is formed at $r_{n u c}$ but can persist at lower relative humidities. Values of $a \leq 1$ would also accommodate cirrus forming at a lower relative humidity due to heterogeneous ice nucleation. The parameter $a$ must range between $r_{s a t} / r_{n u c}$ and unity, since on the one hand supersaturation is observed in the atmosphere and on the other hand cirrus is observed at relative humidities lower than $r_{\text {nuc }}$.

[7] Assuming the uniform PDF of total water with fixed variance, the fraction of the grid box exceeding ice satura- tion or allowing natural cirrus formation, $B_{c c}+c i$ (Figure 1, dashed red curve), is

$$
B_{c c+c i}=\left\{\begin{array}{cl}
\frac{r-r_{c c}}{r_{s a t}-r_{c i}}-b_{c i}\left(1-b_{c i}\right) & : \quad r_{c c} \leq r<r_{*} \\
1 & : \quad r \geq r_{*},
\end{array}\right.
$$

where $r_{*}=r_{s a t}-\left(r_{c i}-r_{c c}\right)^{2} /\left(r_{s a t}-r_{c i}\right)$ (for the derivation and discussion see the auxiliary material). $B_{c c}+c i$ increases linearly with $r$ until clouds are formed at which point it increases with the same slope as $b_{c i}$. The fractional supersaturated area comprises the natural cirrus coverage, $b_{c i}$, and the supersaturated but cloud free area, $B_{c c}$ (Figure 1, solid red curve):

$$
B_{c c}=B_{c c+c i}-b_{c i}
$$

Within $B_{c c}$ contrail cirrus can persist so that it is also called potential contrail cirrus coverage. It can be nonzero in times of zero cirrus coverage consistent with supersaturation that can be observed in cloud-free air. Contrail cirrus can often be observed prior to the onset of natural cirrus. Using observations of supersaturation as a constraint, we find $a=0.9$ to give a realistic $B_{c c}$ (section 3).

[8] Analogous to $r_{c c}$ from equation 2, the critical relative humidity for contrail formation, $r_{c o}$, may be calculated $r_{c o} / r_{c i}=r_{S A C} /\left(a \cdot r_{\text {nuc }}\right)$, with $r_{S A C}$ being the relative humidity over ice at which contrails form during the mixing process between the aircraft jet engine exhaust and the surrounding air. Our potential contrail coverage, $B_{c o}$ (Figure 1, solid green curve), is then defined as in equations 3 and 4 replacing $r_{c c}$ by $r_{c o}$. Since we only discuss contrails that are persistent, $B_{c o}$ is limited to be smaller than $B_{c c}$.

[9] The functional form of our $B_{c o}$ and the critical relative humidity used here differ from the previously used parameterization of $B_{c o}$ [Ponater et al., 2002] (Figure 1, solid blue curve). The old parameterization and the model's cloud coverage are based on different assumptions about the subgrid scale variability of relative humidity and are therefore inconsistent (see auxiliary material). Furthermore, the old parameterization was based on $r_{c o} / r_{c i}=r_{S A C, l}$, with $r_{S A C, l}$ denoting the threshold relative to liquid water and therefore implicitly assumes that natural cirrus form at water saturation. The two parameterizations of $B_{c o}$ differ significantly with the new one allowing a larger (smaller) fraction of contrail coverage at high (low) relative humidities. In the following we analyze a 5-year ECHAM4 integration.

\section{Results}

\subsection{Potential Contrail and Contrail Cirrus Coverage}

[10] North of $40 \mathrm{~N}$ at $300 \mathrm{hPa}$, the SAC is mostly fulfilled when air is supersaturated and hence potential contrail coverage (Figure 2a) and potential contrail cirrus coverage (Figure 2b) are very similar. At $300 \mathrm{hPa}$ potential contrail coverage decreases strongly in the subtropics and is close to zero in the tropics due to warmer temperatures. In contrast, potential contrail cirrus coverage is lowest in the dry subtropics and increases again towards the tropics with maxima in areas of maximum relative humidity. Attainable contrail cirrus coverage may be substantially larger than potential contrail coverage in supersaturated areas, where 

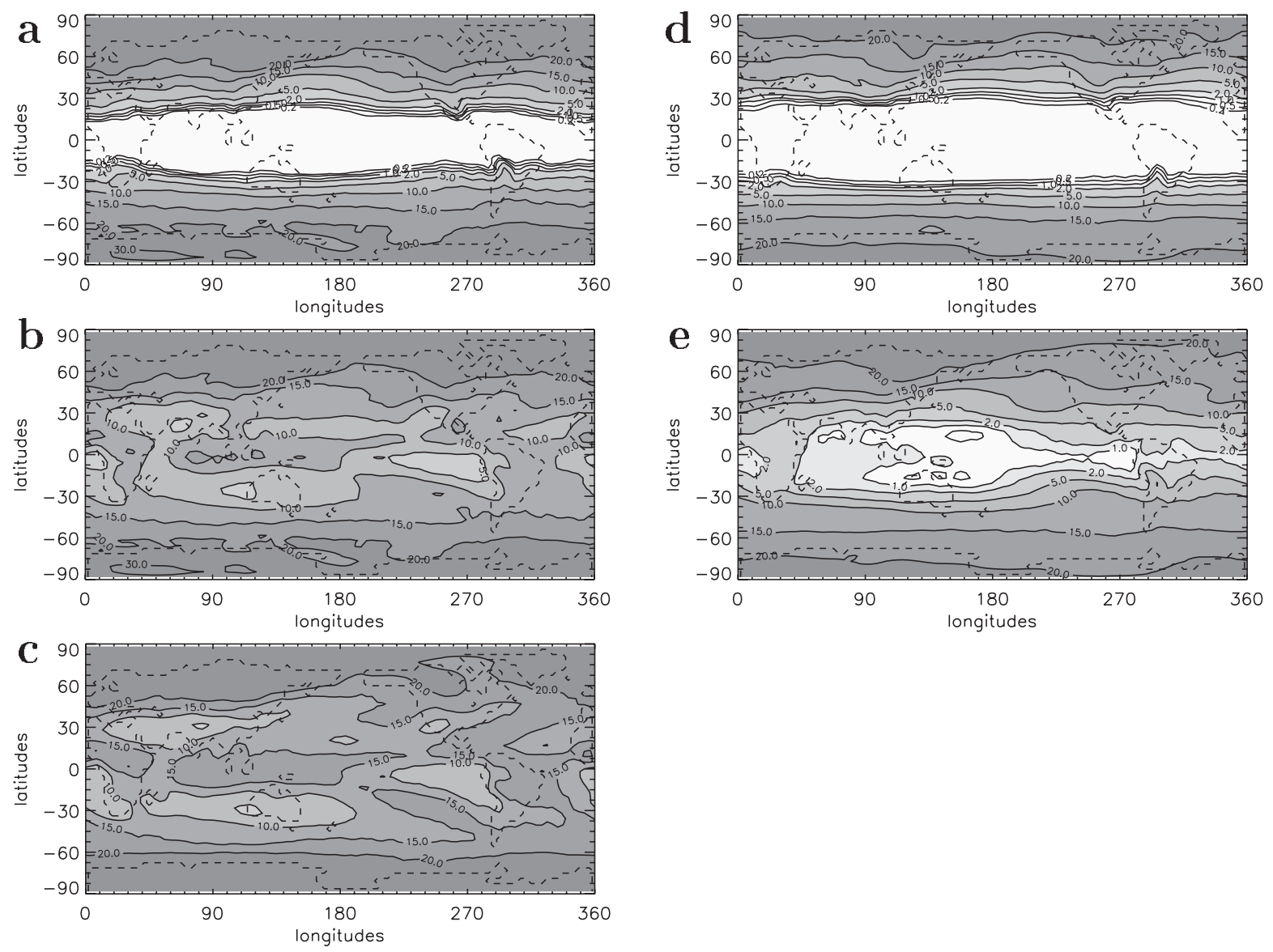

Figure 2. Annual mean (a) potential contrail and (b) potential contrail cirrus coverage at $300 \mathrm{hPa}$ and (c) potential contrail cirrus coverage at $230 \mathrm{hPa}$ as determined consistently with the ECHAM4 natural cloud coverage in \%. Potential contrail coverage at (d) $300 \mathrm{hPa}$ and (e) $230 \mathrm{hPa}$ as calculated according to Ponater et al. [2002] in \%.

the SAC is only intermittently fulfilled, since contrails can spread even when the SAC is not met. In the presence of a large potential contrail coverage gradient, contrails may also be advected into adjoining supersaturated areas not allowing contrail formation. If the former two situations are too infrequently met then potential contrail cirrus coverage is not a measure for maximum attainable contrail cirrus coverage. At $230 \mathrm{hPa}$, temperatures are well below the critical temperature for contrail formation, so that potential (persistent) contrail coverage (not shown) and potential contrail cirrus coverage (Figure 2c) are almost identical except over the Maritime Continent where relative differences are up to $25 \%$.

\subsection{Potential Contrail Cirrus Coverage and Supersaturation}

[11] In Table 1 the tropospheric MOZAIC supersaturation frequency, obtained from measurements onboard commercial airplanes operating mostly over the USA, Europe and the North Atlantic [Gierens et al., 1999], is compared with the fractional potential contrail cirrus coverage at the average flight level of $\sim 230 \mathrm{hPa}$ (tropospheric values only) roughly in the areas where most MOZAIC aircraft fly. Both variables may be interpreted as the probability of an airplane flying within a supersaturated region. Since MOZAIC does not distinguish between in-cloud and clear sky supersaturation and ECHAM does not distinguish between saturated and supersaturated air inside cirrus, a direct comparison may be misleading. Spichtinger et al. [2004] estimate that between $13 \%-33 \%(2 \%-8 \%)$ of supersaturation counts south of $30 \mathrm{~N}$ (north of $30 \mathrm{~N}$ ) represent in-cloud measurements, resulting in a small correction. The parameterization is quite successful at representing the frequency of supersaturation but underestimates the meridional gradient leading to slightly too low (high) values in

Table 1. Frequency of Tropospheric Supersaturation From MOZAIC and AIRS Versus Potential Contrail Cirrus Coverage $B_{c c}$ From ECHAM4 for Different Seasons and Latitude/Longitude Belts $^{\mathrm{a}}$

\begin{tabular}{lcccccccccc}
\hline & \multicolumn{4}{c}{ Midlatitudes } & & \multicolumn{4}{c}{ Tropics } \\
\cline { 2 - 5 } \cline { 7 - 9 } & DJF & MAM & JJA & SON & & DJF & MAM & JJA & SON \\
\hline ECHAM4, 230 hPa & 0.19 & 0.21 & 0.18 & 0.19 & & 0.13 & 0.14 & 0.11 & 0.14 \\
ECHAM4, 275 hPa & 0.18 & 0.18 & 0.17 & 0.17 & & 0.11 & 0.12 & 0.10 & 0.12 \\
MOZAIC, 230 hPa & 0.27 & 0.19 & 0.18 & 0.24 & & 0.13 & 0.10 & 0.10 & 0.10 \\
MOZAIC corrected & 0.26 & 0.18 & 0.17 & 0.23 & & 0.10 & 0.08 & 0.08 & 0.08 \\
AIRS, 250-300 hPa & 0.22 & 0.31 & 0.22 & 0.17 & & 0.02 & 0.02 & 0.02 & 0.02 \\
\hline
\end{tabular}

${ }^{a}$ Midlatitudes: $30 \mathrm{~N}-60 \mathrm{~N} / 95 \mathrm{~W}-35 \mathrm{E}$ and tropics: $30 \mathrm{~N}-30 \mathrm{~S} / 80 \mathrm{~W}-105 \mathrm{E}$. MOZAIC corrected indicates frequencies corrected for in-cloud measurements (in the tropics by $23 \%$ and in the mid latitudes by $5 \%$ ). 

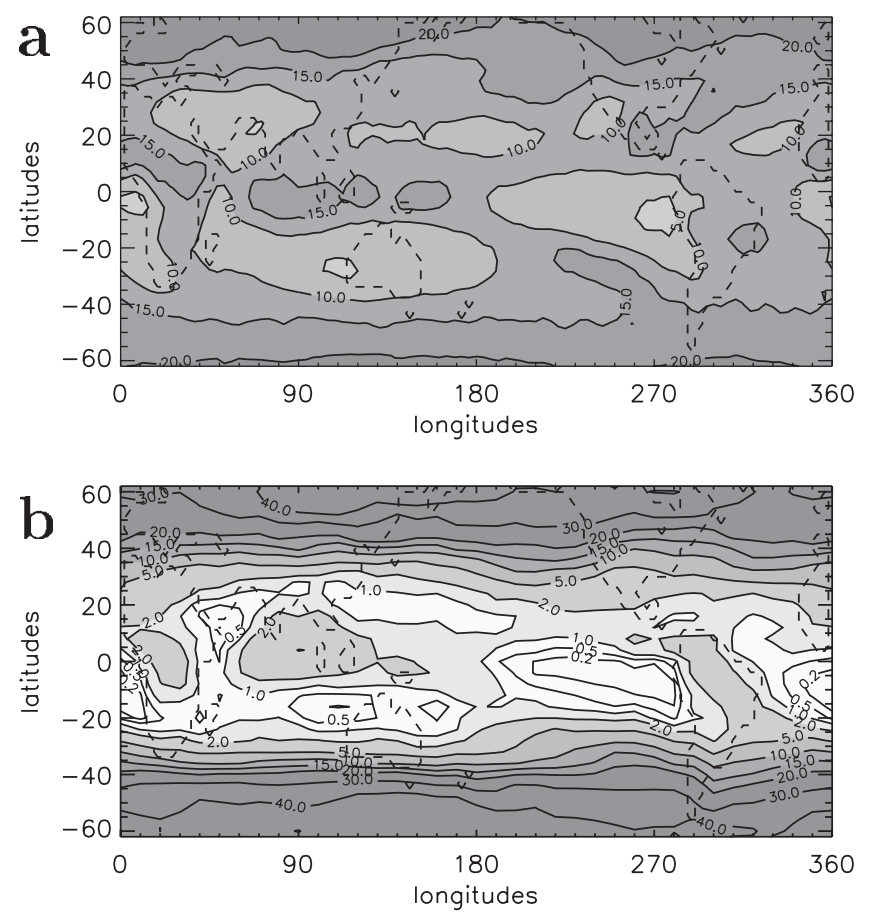

Figure 3. Annual mean tropospheric (a) potential contrail cirrus coverage from ECHAM4 at $275 \mathrm{hPa}$ and (b) frequency of supersaturation from AIRS in $250-300 \mathrm{hPa}$ in $\%$.

the extratropics (tropics). The seasonal cycle in the extratropics is underestimated and slightly shifted, with maxima occurring in spring instead of winter.

[12] Potential contrail cirrus coverage at $300 \mathrm{hPa}$ (tropospheric values only) (Figure 3a) has also been compared to the AIRS tropospheric supersaturation frequency of Gettelman et al. [2006] (Figure 3b). AIRS samples only supersaturation in the presence of less than $70 \%$ cloud coverage. Due to the different horizontal resolutions $\left(3.75^{\circ}\right.$ in ECHAM simulations versus $1^{\circ}$ for AIRS) cloud screening was not applied to the GCM data. The general pattern of supersaturation frequency in the tropics and subtropics is well reproduced by the model. The belt of minimum potential contrail cirrus coverage in the subtropics resembles very much the belt of low supersaturation frequencies from AIRS. In northern summer both fields display a maximum over the tropics close to the monsoon area at about $80 \mathrm{E} / 10 \mathrm{~N}$ (not shown). However, there are also marked differences between the two estimates. Frequency of supersaturation from AIRS data has a larger meridional gradient resulting in larger (lower) values in the extratropics (tropics) than the GCM potential contrail cirrus coverage. Supersaturation frequencies have been averaged in the same areas as above (Table 1) at $250-300 \mathrm{hPa}$ rather than $200-250 \mathrm{hPa}$, as data in the upper levels may be contaminated by stratospheric humidity values due to the broad vertical weighting function of AIRS $(1-3 \mathrm{~km})$ causing lower frequencies of supersaturation especially in the extratropics. Area averaged frequencies in the tropics are lower than either the MOZAIC estimate and the GCM estimate (Table 1). This may be expected since tropical supersaturation often occurs near convection which AIRS does not sample well. The AIRS seasonal cycle shows, contrary to MOZAIC, a pronounced extratropical maximum in spring.

[13] It is difficult to obtain any firm conclusions from the comparison with the AIRS data considering that derived relative humidity suffers from a large number of uncertainties [Read et al., 2007] so that absolute values of supersaturation must be regarded highly uncertain. MOZAIC and AIRS estimates suffer from sparse and seasonally varying spatial sampling and coarse vertical resolution, respectively. Both AIRS and MOZAIC data sets are short and may not represent climatological values. These temporal and spatial sampling issues are likely to be the reason for the differences in the AIRS and MOZAIC seasonal cycle of extratropical supersaturation. Regardless, the agreement in the patterns of supersaturation frequency and potential contrail cirrus coverage is very encouraging. Note that given the uncertainties in AIRS relative humidity retrievals [Gettleman et al., 2006] and in MOZAIC data, due to cloud contamination, AIRS and MOZAIC results may not be inconsistent.

\subsection{Potential Contrail Coverage}

[14] Comparing our potential contrail coverage with earlier estimates of Ponater et al. [2002] (Figures 2d and 2e) reveals large differences in the subtropics and extratropics. At $300 \mathrm{hPa}$ (Figures 2a and 2d) the patterns look fairly similar with the maximum of the meridional gradient being moved towards the tropics relative to the older estimates. At $230 \mathrm{hPa}$, a level at which temperatures are well below the temperature threshold for contrail formation, potential contrail coverage (not shown) is nearly identical to potential contrail cirrus coverage (Figure 2c). Minima of potential contrail coverage as calculated from equations 3 and 4 are situated in the subtropics in the same places as the minima of supersaturation frequency as inferred from AIRS (Figure 3b). In contrast, the older parameterization [Ponater et al., 2002] resulted in minima that were situated in the tropics (Figure 2e). Differences between the two approaches are therefore largest in the tropics. Over the Maritime Continent, an area where air traffic is projected to grow strongly, potential contrail coverage is larger (up to 13\%) than according to previous estimates.

\section{Summary}

[15] Potential contrail coverage is not a measure for maximum attainable coverage due to contrail cirrus, since contrail cirrus can spread in supersaturated areas when the SAC is not met. Neither is potential contrail cirrus coverage (the cloud free supersaturated area) a measure for the maximum attainable coverage since formation conditions may be too infrequently met and only few contrail cirrus may be advected into those areas. The difference between both potential coverages describes the areas in which maximum attainable contrail cirrus coverage may exceed potential contrail coverage. Potential contrail cirrus coverage is larger than potential contrail coverage especially at lower levels and in the tropics.

[16] The supersaturation frequency parameterized in ECHAM4 compares well with AIRS satellite retrievals in terms of the large scale pattern of supersaturation frequency and with MOZAIC in situ data in terms of overall values. 
We judge our estimate of potential contrail cirrus coverage to be good as far as any firm judgment is possible. The physically consistent parameterization of potential contrail coverage yields larger values than the old formulation especially when relative humidity is high. Changes are largest in the tropics especially over the Maritime Continent. The large scale pattern of potential contrail coverage agrees better with AIRS observations when using the new instead of the old formulation.

[17] In principle it would be desirable to calculate contrail and cirrus coverages from explicitly simulated supersaturation, however, validating supersaturation and high cloud coverages is difficult due to a large number of uncertainties in the observational data sets. Meanwhile our parameterization will result in improved estimates of line-shaped contrail coverage and will enable the estimation of contrail cirrus coverage. More effort should be also put into assessing the performance of the cloud scheme using different observational cirrus data sets.

[18] Acknowledgments. This work was carried out within the DLR projects PAZI-2 and CATS. MOZAIC has been sponsored since 1994 by the European Commission. National Center for Atmospheric Research is supported by the United States National Science Foundation.

\section{References}

Chen, C.-T., and E. Roeckner (1997), Cloud simulations with the Max Planck Institute for Meteorology general circulation model ECHAM4 and comparison with observations, J. Geophys. Res., 102, 9335-9350.

Gettelman, A., E. J. Fetzer, A. Eldering, and F. W. Irion (2006), The global distribution of supersaturation in the upper troposphere from the Atmospheric Infrared Sounder, J. Clim., 19, 6089-6103.

Gierens, K., U. Schumann, M. Helten, H. G. J. Smit, and A. Marenco (1999), A distribution law for relative humidity in the upper troposphere and lower stratosphere derived from three years of MOZAIC measurements, Ann. Geophys., 17, 1218-1226.

Gregory, D., D. Wilson, and A. Bushell (2002), Insights into cloud parametrization provided by a prognostic approach, Q. J. R. Meteorol. Soc., $128,1485-1504$.

Jensen, E. J., O. B. Toon, S. A. Vay, J. Ovarlez, R. May, T. P. Bui, C. H. Twohy, B. W. Gandrud, R. F. Pueschel, and U. Schumann (2001), Prevalence of ice-supersaturated regions in the upper troposphere: Impli- cations for optically thin ice cloud formation, J. Geophys. Res., 106, $17,253-17,266$.

Koop, T. (2004), Homogeneous nucleation in water and aqueous systems, Z. Phys. Chem., 218, 1231-1258.

Land, C., J. Feichter, and R. Sausen (2002), Impact of the vertical resolution on the transport of passive tracers in the ECHAM4 model, Tellus, Ser. B, 54, 344-360.

Minnis, P., U. Schumann, D. R. Doelling, K. Gierens, and D. W. Fahey (1999), Global distribution of contrail radiative forcing, Geophys. Res. Lett., 26, 1853-1856.

Myhre, G., and F. Stordal (2001), On the tradeoff of the solar and thermal infrared impact of contrails, Geophys. Res. Lett., 28, 3119-3122.

Ponater, M., S. Marquart, and R. Sausen (2002), Contrails in a comprehensive global climate model: Parameterization and radiative forcing results, J. Geophys. Res., 107(D13), 4164, doi:10.1029/2001JD000429.

Rädel, G., and K. P. Shine (2008), Influence of aircraft cruise altitudes on radiative forcing by persistent contrails, J. Geophys. Res., 113, D07105, doi:10.1029/2007JD009117.

Read, W. G., et al. (2007), Aura Microwave Limb Sounder upper tropospheric and lower stratospheric $\mathrm{H}_{2} \mathrm{O}$ and relative humidity with respect to ice validation, J. Geophys. Res., 112, D24S35, doi:10.1029/ 2007JD008752.

Roeckner, E., L. Bengtsson, J. Feichter, J. Lelieveld, and H. Rodhe (1999), Transient climate change simulations with a coupled atmosphere-ocean GCM including the tropospheric sulfur cycle, J. Clim., 12, 3004-3032.

Sausen, R., K. Gierens, M. Ponater, and U. Schumann (1998), A diagnostic study of the global distribution of contrails. Part I: Present day climate, Theor. Appl. Clim., 61, 127-141.

Sausen, R., et al. (2005), Aviation radiative forcing in 2000: An update on IPCC (1999), Meteorol. Z., 14, 555-561.

Schumann, U. (1996), On conditions for contrail formation from aircraft exhausts, Meteorol. Z., 5, 4-23.

Spichtinger, P., K. Gierens, H. G. J. Smit, J. Ovarlez, and J.-F. Gayet (2004), On the distribution of relative humidity in cirrus clouds, Atmos. Chem. Phys., 4, 639-647.

Stuber, N., and P. Forster (2007), The impact of diurnal variations of air traffic on contrail radiative forcing, Atmos. Chem. Phys., 7, 3153-3162. Sundqvist, H. (1978), A parameterization scheme for non-convective condensation including prediction of cloud water content, Q.J.R. Meteorol. Soc., 104, 677-690.

U. Burkhardt, K. Gierens, B. Kärcher, and M. Ponater, Deutsches Zentrum für Luft- und Raumfahrt, Institut für Physik der Atmosphäre, D-82234 Oberpfaffenhofen, Germany. (ulrike.burkhardt@dlr.de)

A. Gettelman, National Center for Atmospheric Research, Boulder, CO 80305, USA. 